\title{
Evaluating the cleavage efficacy of CRISPR-Cas9 sgRNAs targeting ineffective regions of Arabidopsis thaliana genome
}

\author{
Afsheen Malik ${ }^{1}$, Alvina Gul ${ }^{\text {Corresp., }}{ }^{1}$, Faiza Munir ${ }^{1}$, Rabia Amir ${ }^{1}$, Hadi Alipour ${ }^{2}$, Mustafeez Mujtaba Babar ${ }^{3}$, Syeda \\ Marriam Bakhtiar ${ }^{4}$, Rehan Zafar Paracha ${ }^{5}$, Zoya Khalid ${ }^{6}$, Muhammad Qasim Hayat ${ }^{1}$ \\ 1 Department of Plant Biotechnology, Atta-ur-Rahman School of Applied Biosciences, National University of Sciences and Technology, Islamabad, Pakistan \\ Department of Plant Production and Genetics, Faculty of Agriculture and Natural Resources, Urmia University, Urmia, Iran \\ 3 Shifa College for Pharmaceutical Sciences, Shifa Tameer-e-Millat University, Islamabad, Pakistan \\ 4 Department of Bioinformatics and Biosciences, Capital University of Science and Technology, Islamabad, Pakistan \\ 5 Research Center for Modeling and Simulation, National University of Sciences and Technology, Islamabad, Pakistan \\ ${ }^{6}$ Computational Biology Research Lab, Department of Computer Science, National University of Computer and Emerging Sciences-FAST, Islamabad, \\ Pakistan \\ Corresponding Author: Alvina Gul \\ Email address: alvina_gul@asab.nust.edu.pk
}

The CRISPR-Cas9 system has recently evolved as a powerful mutagenic tool for targeted genome editing. The impeccable functioning of the system depends on the optimal design of single guide RNAs (sgRNAs) that mainly involves sgRNA specificity and on-target cleavage efficacy. Several research groups have designed algorithms and models, trained on mammalian genomes, for predicting sgRNAs cleavage efficacy. These models are also implemented in most plant sgRNA design tools due to the lack of on-target cleavage efficacy studies in plants. However, one of the major drawbacks is that almost all of these models are biased for considering only coding regions of the DNA while excluding ineffective regions, which are of immense importance in functional genomics studies especially for plants, thus making prediction less reliable. In the present study, we evaluate the on-target cleavage efficacy of experimentally validated sgRNAs designed against diverse ineffective regions of Arabidopsis thaliana genome using various statistical tests. We show that nucleotide preference in protospacer adjacent motif (PAM) proximal region, GC content in the PAM proximal seed region, intact RAR and $3^{\text {rd }}$ stem loop structures, and free accessibility of nucleotides in seed and tracrRNA regions of sgRNAs are important determinants associated with their high on-target cleavage efficacy. Thus, our study describes the features important for plant sgRNAs high on-target cleavage efficacy against previously unexplored ineffective genomic regions. Moreover, it suggests the need of developing an elaborative plant-specific sgRNA design model considering the entire genomic landscape including ineffective regions for enabling highly efficient genome editing without wasting time and experimental resources. 
1 Evaluating the cleavage efficacy of CRISPR-Cas9 sgRNAs targeting ineffective regions of 2 Arabidopsis thaliana genome

3

4

Afsheen Malik ${ }^{1}$, Alvina Gul ${ }^{1}$, Faiza Munir ${ }^{1}$, Rabia Amir ${ }^{1}$, Hadi Alipour ${ }^{2}$, Mustafeez Mujtaba Babar $^{3}$, Syeda Marriam Bakhtiar ${ }^{4}$, Rehan Zafar Paracha ${ }^{5}$, Zoya Khalid ${ }^{6}$, Muhammad Qasim Hayat $^{1}$

${ }^{1}$ Department of Plant Biotechnology, Atta-ur-Rahman School of Applied Biosciences, National University of Sciences and Technology, Islamabad, Pakistan

${ }^{2}$ Department of Plant Production and Genetics, Faculty of Agriculture and Natural Resources, Urmia University, Urmia, Iran

${ }^{3}$ Shifa College for Pharmaceutical Sciences, Shifa Tameer-e-Millat University, Islamabad, Pakistan

${ }^{4}$ Department of Bioinformatics and Biosciences, Capital University of Science and Technology, Islamabad, Pakistan

${ }^{5}$ Research Center for Modeling and Simulation, National University of Sciences and Technology, Islamabad, Pakistan

${ }^{6}$ Computational Biology Research Lab, Department of Computer Science, National University of Computer and Emerging Sciences-FAST, Islamabad, Pakistan

Corresponding Author:

Alvina Gul

alvina_gul@asab.nust.edu.pk 
44

45

46

47

48

49

50

51

52

53

54

55

56

57

58

59

60

61

62

63

64

65

66

67

68

69

70

71

72

73

74

75

76

77

78

79

80

81

82

83

84

85

86

87

\section{Abstract}

The CRISPR-Cas9 system has recently evolved as a powerful mutagenic tool for targeted genome editing. The impeccable functioning of the system depends on the optimal design of single guide RNAs (sgRNAs) that mainly involves sgRNA specificity and on-target cleavage efficacy. Several research groups have designed algorithms and models, trained on mammalian genomes, for predicting sgRNAs cleavage efficacy. These models are also implemented in most plant sgRNA design tools due to the lack of on-target cleavage efficacy studies in plants. However, one of the major drawbacks is that almost all of these models are biased for considering only coding regions of the DNA while excluding ineffective regions, which are of immense importance in functional genomics studies especially for plants, thus making prediction less reliable. In the present study, we evaluate the on-target cleavage efficacy of experimentally validated sgRNAs designed against diverse ineffective regions of Arabidopsis thaliana genome using various statistical tests. We show that nucleotide preference in protospacer adjacent motif (PAM) proximal region, GC content in the PAM proximal seed region, intact RAR and $3^{\text {rd }}$ stem loop structures, and free accessibility of nucleotides in seed and tracrRNA regions of sgRNAs are important determinants associated with their high on-target cleavage efficacy. Thus, our study describes the features important for plant sgRNAs high on-target cleavage efficacy against previously unexplored ineffective genomic regions. Moreover, it suggests the need of developing an elaborative plant-specific sgRNA design model considering the entire genomic landscape including ineffective regions for enabling highly efficient genome editing without wasting time and experimental resources.

\section{Introduction}

Traditionally, scientists have been using various physical, chemical and biological techniques like irradiation, chemical and insertional mutagenesis either for incorporating traits of agricultural importance in crop plants or studying and deciphering important biological mechanisms in model plants (Wu et al., 2005; Tadege et al., 2009; Oladosu et al., 2016). However, the potential disadvantages associated with these traditional techniques are that all of these methods induce mutations in genome randomly that have a high tendency of producing undesired mutations and phenotypes (Shalem, Sanjana \& Zhang, 2015; Chaudhary et al., 2019). Moreover, search for desired mutations requires screening bulk populations often accompanied by constructing mapping population and map-based cloning which are laborious, costly, and time-consuming processes (Gilchrist \& Haughn, 2010; Lee, Gould \& Stinchcombe, 2014). Thus, the development of techniques that can transform plant genetics and improve crop plants by overcoming these limitations are highly desired. The discovery of designer nucleases, which can be engineered for targeted genome editing, has emerged as a powerful tool over current

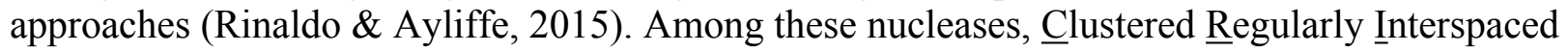

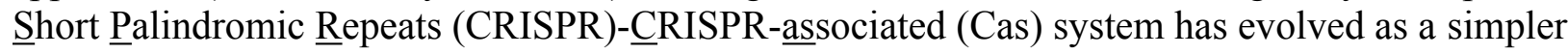
and efficient mutagenic tool that can be used in diverse organisms including plants (Sander \& Joung, 2014; Hussain, Lucas \& Budak, 2018). CRISPR-Cas system works as a part of the bacterial or archaeal adaptive immune system where it safeguards them from invading foreign DNA molecules (Barrangou et al., 2007; Wiedenheft, Sternberg \& Doudna, 2012). The standard CRISPR-Cas9 system, derived from Streptococcus pyogenes, is widely adopted for mediating targeted genome editing due to its relative simplicity. In this regard, a major breakthrough occurred when synthetic chimera of CRISPR RNA (crRNA) and trans-activating crRNA 
88 (tracrRNA) moieties known as single guide RNA (sgRNA) was generated that successfully 89 guided the Cas9 to specific sites in the genome for targeted editing (Jinek et al., 2012). The 2090 nucleotide spacer sequence (denoted as gRNA in the current study) at the $5^{\prime}$ end of the sgRNA

91

92

93

94

95

96

97

98

99

100

101

102

103

104

105

106

107

108

109

110

111

112

113

114

115

116

117

118

119

120

121

122

123

124

125

126

127

128

129

130

131

132

133 directs the Cas 9 protein to the complementary target sequence marked by NGG protospacer adjacent motif (PAM) present downstream of it for inducing double stranded breaks (DSBs) and also determines the specificity and cleavage efficacy of Cas9 endonuclease (Jinek et al., 2012; Wong, Liu \& Wang, 2015).

Despite the simplicity and robustness of the system, the sgRNA specificity and on-target cleavage efficacy are the major concerns in CRISPR-Cas9 mediated genome editing. Different computational tools have been developed for determining the sgRNAs specificities (reviewed in Liu, Zhang \& Zhang, 2019). Moreover, double nicking and transcriptional activation domainbased studies have been shown promising for improving sgRNA on-target specificity (Ran et al., 2013; Mali et al., 2013). Determining the sgRNA specificity and off-target prediction for mammalian systems is very important compared to plants, as the backcrossing can easily alleviate off-target effects in plants (Kim, Alptekin \& Budak, 2018; Naim et al., 2020). The second important factor determining the Cas9 effectiveness that impacts both mammalian and plant systems is the on-target cleavage efficacy of sgRNAs. Recently, sgRNAs cleavage efficacies have been realized, as several groups have identified various sequence and structural features of sgRNAs affecting their on-target cleavage efficacy and have developed models and algorithms, which are now incorporated in different computational tools for designing optimum sgRNAs (Cong et al., 2013; Doench et al., 2014; Heigwer, Kerr \& Boutros, 2014; Wang et al., 2014; Wu et al., 2014; Xie, Zhang \& Yang, 2014; Chari et al., 2015; Fusi et al., 2015; Housden et al., 2015; Moreno-Mateos et al., 2015; Wong, Liu \& Wang, 2015; Xu et al., 2015; Doench et al., 2016; Liang et al., 2016; Cao et al., 2017; Chari et al., 2017; Labuhn et al., 2018; Mendoza \& Trinh, 2018; Labun et al., 2019). Despite all these advancements, some major issues are associated with almost all of these models. For instance, models are trained on datasets derived from few mammalian systems. Datasets are derived from coding regions of genomes that add biasness to the analysis and models. Moreover, not all of these tools have user-friendly interfaces and there is a lack of consistency among the outputs, which raises reliability concerns (Liu, Zhang \& Zhang, 2019). While on the other side, in the plant sciences community, the problem is more complicated by the fact that only some plant-specific sgRNA prediction tools are available that offer sgRNA design for a limited number of plant genomes (Table 1). Most of the plant sgRNA design tools use mammalian systems derived models for off-target predictions and determining on-target efficacy, thus giving rise to inconsistency and discrepancies between predicted and observed in vivo CRISPR-Cas9 working. Furthermore, studies evaluating the ontarget cleavage efficacies are lacking in plants (Liang et al., 2016; Naim et al., 2020). Thus, all these factors demand further work in these directions.

The Non-coding regions of DNA not only maintain the structure of chromatin but also harbor important regulatory elements (Böhmdorfer \& Wierzbicki, 2015; Shanmugam, Nagarajan \& Pramanayagam, 2017). Almost all sgRNA design models and algorithms are trained on datasets that exclude non-coding regions from analysis because of their potential to give rise to noneffective sgRNAs despite realizing the importance of these regions. Therefore, most plant functional genomics studies that require the deletion of large chromosomal parts or deciphering the functional role of regulatory elements often face failure because of the inability of sgRNA 
134 design tools for predicting efficient sgRNAs against these regions (Durr et al., 2018). Thus, for

135

136

137

138

139

140

141

142

143

144

145

146

147

148

149

150

151

152

153

154

155

156

157

158

159

160

161

162

163

164

165

166

167

168

169

170

171

172

173

174

175

the successful application of CRISPR-Cas9 technology against non-coding regions, consideration of these regions is of immense importance while orchestrating the models.

In the present study, we target DNA regions that are excluded from sgRNA design model (Doench et al., 2014) along with other non-coding regions for determining the various sequence and structural features of sgRNAs potentially associated with their high on-target cleavage efficacies against these regions. These regions include 5' untranslated regions (5' UTRs), 3' untranslated regions ( $3^{\prime}$ UTRs), introns, area near $\mathrm{N}$ - and C-terminal regions, which were reported as "broadly ineffective target regions" for giving rise to ineffective sgRNAs (Doench et al., 2014), long non-coding RNAs (lncRNAs) and intergenic regions (hereafter all these regions will be referred as ineffective regions collectively). For this purpose, we analyze the publicly available and in vivo validated plant sgRNAs data using different statistical tests. We show that nucleotide preference at position near PAM proximal region, GC content in PAM proximal seed region, intact RAR and $3^{\text {rd }}$ stem loop secondary structures, and free accessibility of nucleotides in seed region and tracrRNA region of sgRNAs are the most important factors associated with sgRNAs high on-target cleavage efficacy against ineffective regions of $A$. thaliana genome.

\section{Materials \& Methods}

\section{Retrieval of gRNA sequences}

A total of 106 gRNA sequences targeting 53 loci located on different regions of all 5 chromosomes of $A$. thaliana were retrieved from a study carried by $\mathrm{Wu}$ et al. (2018). To maintain uniformity and to minimize the possible effects of the backbone/scaffolding region and/or other components (Hsu et al., 2013; Bortesi et al., 2016), we selected gRNAs from a single study. The target site locations of these gRNAs were determined using the Seqviewer tool available at The Arabidopsis Information Resource (TAIR) database (https://www.arabidopsis.org/). The target sites for these gRNAs were located mainly in regions like 5' UTRs, 3' UTRs, introns, intron-exon junctions, near C- and N-terminal ends, exons of either target genes or flanking genes, intergenic regions, and long non-coding RNAs (lncRNAs). The gRNAs were selected based on their target gene(s) knockout ability. The redundant gRNAs and those whose target sites could not qualify as ineffective regions were removed from the dataset. Based on the selection criteria, a total of 58 gRNAs $(62 \%)$ were determined as highly efficient and contained gRNAs of two different lengths i.e., $19 \mathrm{bp}$ and $20 \mathrm{bp}$ (Fu et al., 2014). The base composition was determined using the WebLogo server (https://weblogo.berkeley.edu/logo.cgi), while the observed deletion frequencies for the target genes were taken as cleavage efficiencies of their respective gRNAs.

\section{Secondary structure prediction and statistical analysis}

The prediction of secondary structures of gRNAs and sgRNAs (containing gRNA and scaffolding region) were carried out using RNAfold web server (http://rna.tbi.univie.ac.at/cgibin/RNAWebSuite/RNAfold.cgi) available with Vienna RNA software package (Hofacker, 2003) accessible at (http://rna.tbi.univie.ac.at/). The software predicts RNA secondary structures based on minimum free energy (MFE) using the Zuker and Stiegler algorithm (Zuker \& Stiegler, 1981), whereas base pairing probabilities are calculated utilizing the partition algorithm of John

Peer) reviewing PDF | (2020:10:53534:2:0:NEW 8 Mar 2021) 
176

177

178

179

180

181

182

183

184

185

186

187

188

189

190

191

192

193

194

195

196

197

198

199

200

201

202

203

204

205

206

207

208

209

210

211

212

213

214

215

216

217

218

McCaskill (McCaskill, 1990). Before secondary structure prediction, an additional "G" used for enhancing transcription from U6 promoter (Wu et al., 2018) was appended to the sequences. Different statistical tests like Chi-Square, Kruskal-Wallis, and Wilcoxon were employed for determining the features significantly associated with gRNAs high on-target cleavage efficacies, whereas Spearman's rho and Pearson correlation tests were used for inferring the relationship or association of features. The level of significance was taken as $<0.05$. Chi-Square tests were applied using MS Excel, while all other tests were performed by SPSS software (http://www.brothersoft.com/ibm-spss-statistics-469577.html). The boxplots were drawn using ggplot2, dplyr, and ggpubr packages in RStudio software.

\section{Results}

\section{Sequence analysis of gRNAs}

The effectiveness of gRNAs in causing on-target editing is of paramount importance in CRISPRCas9 mediating genome editing. For determining sgRNAs various features responsible for their high on-target cleavage efficiency, we selected experimentally validated highly efficient gRNAs targeting several ineffective regions of DNA. The selected gRNAs along with their target genes IDs, target site sequences, PAM sequences, strand localization, target site annotation, gRNA sequences, GC content, sgRNA sequences, gRNA, and sgRNA secondary structures with their corresponding MFE values are mentioned in Table S1. The secondary structure of sgRNAs used in the study is shown in Fig. 1. For finding significant features associated with gRNAs high ontarget cleavage efficacy, we applied various statistical tests. First, the nucleotide base preference of gRNAs was determined to see if nucleotide base preference is responsible for their on-target cleavage efficacy. Sequence logo created for this purpose revealed a high frequency of thymine at positions $1,3,5,18$, and 19 , whereas guanine at position 20 along with the frequency change for other nucleotide bases (Fig. 2). Next, we wanted to know if these changes in base frequencies at specific positions have some statistical significance or occurred by chance, so we constructed a frequency table for each position and applied the Chi-Square test (Table 2). The Chi-Square test analysis revealed a significant change in base frequency at position 19 ( $p$-value $=8.6 \mathrm{E}-03)$. Next, to analyze whether GC content has any impact on activities, we first determined the GC content percentage of full-length gRNAs. Though we could not find any significant change overall ( $p$-value $=1.2 \mathrm{E}-01)$ but we observed that gRNAs with GC content in ranges of $0-40 \%$ and $41-55 \%$ showed better cleavage activity as compared to gRNAs with GC content $56-100 \%$ (Fig. 3A). As we observed that GC content variation tends to impact the activity, therefore, next we divided the gRNA sequences into sections of different lengths while moving from PAM proximal region to distal region to see if GC content variation only affects the gRNA subregions. Since the seed region of gRNA is imperative for the activity, therefore, we calculated the GC content percentage of the PAM proximal seed region (1-12 nt) and the PAM distal region (13-20 nt). In the case of the PAM proximal seed region (1-12 nt), the GC content positively and significantly impacted the cleavage efficacy $(p$-value $=3.2 \mathrm{E}-02)$. The comparison of groups demonstrated that significant difference is associated with medium (35-55\%) and high (56$100 \%$ ) GC content groups and cleavage activities remarkably decreased with increasing GC content (Fig. 3B). Regarding PAM distal region (13-20 nt), an overall significant change could not be found $(p$-value $=4.3 \mathrm{E}-01)$. However, the cleavage efficacy was positively influenced by increased GC content i.e., $56-100 \%$ and $31-55 \%>0-30 \%$ (Fig. 3C). Next, we performed

Peer] reviewing PDF | (2020:10:53534:2:0:NEW 8 Mar 2021) 
219 complete tilling with a window size of 5 nucleotides while moving one nucleotide from PAM

220

221

222

223

224

225

226

227

228

229

230

231

232

233

234

235

236

237

238

239

240

241

242

243

244

245

246

247

248

249

250

251

252

253

254

255

256

257

258

259

260

261

262 proximal to distal region across the entire gRNA sequence to see if narrowing down can provide further insight. The 5 nucleotide-wide window tilling could not reveal any significant difference overall ( $p$-values range $=0.88-0.06$; Figs. S1A-S1P). Further, we determined the impact of the same di and tri contiguous bases on gRNAs on-target editing efficiency. We could not observe any significant effect of dinuceosides ( $p$-values range $=0.97-0.2)$ and trinucleosides $(p$-values range $=1.0-0.45)$ on the activity. However, we found that gRNAs with two dinucleosides AA and TT (Figs. 4A-4B) showed non-significantly ( $p$-values $=4.4 \mathrm{E}-01$ and 2.0E-01, respectively) enhanced activity compared to gRNAs with two GG and CC where their depletion resulted in more efficient gRNAs (Figs. 4C-4D). In the case of trinucleosides, gRNAs with depleted trinucloesides (i.e., AAA, GGG, and CCC) positively influenced the cleavage efficacy except for those gRNAs where the presence of one trinucleoside (TTT) or the absence did not make any difference (Fig. 5). Besides determining gRNAs sequence features, we wanted to ascertain if PAM variable nucleotide (VN) and gRNA target DNA strand have any impact on cleavage efficacy. Our analysis showed no significant influence of these features on gRNA cleavage activity (Figs. 6A-6B).

\section{Structural features analysis}

We manually analyzed sgRNAs secondary structures for the determination of the differences in availability of bases at seed regions (18-20 bp in case of $20 \mathrm{bp}$ long gRNAs and 19-21 bp in case of $21 \mathrm{bp}$ long gRNAs) and tracrRNA regions (59-61 bp and 60-62 bp in case of 20 and $21 \mathrm{bp}$ long gRNAs, respectively) that can contribute to the on-target cleavage efficacy. We found significant changes at positions $19(p$-value $=6.3 \mathrm{E}-06), 20(p$-value $=2.4 \mathrm{E}-03), 59(p$-value $=$ $4.0 \mathrm{E}-04), 60(p$-value $=3.5 \mathrm{E}-06), 61(p$-value $=1.7 \mathrm{E}-05)$, and $62(p$-value $=5.3 \mathrm{E}-04)($ Table 3$)$. Also, the secondary structures of sgRNAs were analyzed for the presence of intact stem loop elements. We found that stem loop 2 was absent, stem loop RAR and stem loop 3 were present in every sgRNA sequence, while only $5 \%$ of the sgRNAs had stem loop 1 structure. Additionally, to determine the influence of secondary structure stability of gRNAs on their cleavage efficacy, we divided the gRNA secondary structure $\Delta \mathrm{G}$ values into different stability groups. However, we could not find any statistically significant difference that can relate the gRNA structure internalization stabilities with their efficacies ( $p$-value $=2.8 \mathrm{E}-01$; Fig. 7$)$.

\section{Association of cleavage efficacy with sgRNAs features}

We carried out Spearman's rho correlation and Pearson correlation tests for determining the association of gRNAs full-length GC content, $\Delta \mathrm{G}$ of gRNAs, and $\Delta \mathrm{G}$ of $\operatorname{sgRNAs}$ with cleavage efficacies, respectively. In the case of GC content, a negative and a very weak association was seen with cleavage efficacy $(r=-0.123)$. For $g R N A s \Delta G$ and cleavage efficiency, a positive and a very weak relationship was observed $(r=0.089)$, while in the case of $\operatorname{sgRNA} \Delta \mathrm{G}$, a positive and a very weak association with cleavage efficacy was observed $(r=0.194)$. However, in all the aforementioned cases the statistically significant relationship could not be observed between the variables ( $p$-values $=3.91 \mathrm{E}-01,5.33 \mathrm{E}-01$, and $1.71 \mathrm{E}-01$, respectively).

\section{Discussion}

The current study identifies various sequence and structural features of sgRNAs as important determinants of their high on-target cleavage efficacy against ineffective regions of genomic 
263 DNA that are of immense importance in functional genomics studies. Keeping in mind the 264 importance of ineffective regions, the study targets DNA regions that are excluded from the 265 sgRNA design model (Doench et al., 2014) along with other non-coding genomic regions for 266 determining the various sequence and structural features that affect sgRNAs on-target cleavage sgRNAs, we used publicly available and in vivo validated plant sgRNAs targeting various ineffective regions. Next, to establish the criteria, which can demonstrate their high on-target cleavage efficacies, we applied different statistical tests. The analysis revealed a statistically significant difference at position 19, which constitutes the $3^{\prime}$ end of gRNAs. The position 19 is present adjacent to PAM in $19 \mathrm{bp}$ long sgRNAs and agrees with previous observations (Doench et al., 2014; Wang et al., 2014; Xu et al., 2015; Liu et al., 2016). However, in contrast to previous observations that reported guanine or cytosine as a preferred base at a position adjacent to PAM, our study showed thymine is dominating at this position, while at other positions we did not observe any significant change (Table 2). The dominance of thymine reflects the AT-rich nature of non-coding regions. In 20 bp long gRNAs, we could not find any significant change at position 20, adjacent to PAM, which might be due to the absence of data and/or their small sample size at this position. The gRNA GC content was shown to have an effect on sgRNAs activities with low or high GC content resulting in the generation of inefficient sgRNAs (Doench et al., 2014). Our results showed no statistically significant difference in the GC content of fulllength gRNAs (1-20 nt) (Fig. 3A). However, the analysis of GC content of gRNAs split sections showed that the GC content of PAM proximal seed region (1-12 nt) impacts the cleavage efficacy significantly and increasing GC content significantly decreases gRNAs activity (Fig. 3B) and is in disagreement with the former studies that could not find GC content significant impact in this region (Ren et al., 2014; Labuhn et al., 2018). The presence of the same contiguous bases (TTT, GGG, and GG) may interfere with gRNA transcription or affect their editing efficacies (Wong, Liu \& Wang, 2015). The analysis of our dataset could not reveal any significant correlation of di- and trinucleosides with the efficacy (Figs. 4 and 5), however the observed non-significant increase in gRNAs efficacy with dinucleosides AA, TT, and trinucleosides TTT seems associated with the nature of non-coding regions. In our dataset, the analysis of PAM variable nucleotide and target DNA strand, taken as a function of gRNAs activity, showed no statistically significant impact on their cleavage efficacy (Figs. 6A-6B), which were in contrast to the previous observations (Doench et al., 2014; Wang et al., 2014). The free accessibility of the last three bases of the seed region and the first three bases (AAG) of the tracrRNA region is imperative for on-target cleavage efficiency (Wong, Liu \& Wang, 2015). Our results are in agreement with the aforementioned observations as we found significant differences at these positions (Table 3). Different stem loop elements in secondary structures like RAR, $2^{\text {nd }}$ and $3^{\text {rd }}$ stem loops were shown to be associated with plant sgRNAs on-target efficiency (Ma et al., 2015; Liang et al., 2016). The results showed that the presence of intact RAR and $3^{\text {rd }}$ stem loop structures are important for their on-target cleavage efficacy and the absence of $2^{\text {nd }}$ stem loop element indicates that this secondary structural element does not have any impact on their cleavage efficacy against ineffective genomic regions. Previous studies showed that energetically stable gRNAs secondary structures are responsible for cleavage inefficiencies (Wong, Liu \& Wang, 2015; Thyme et al., 2016, Jensen et al., 2017), which were in contrast to our results, as we could not find statistically meaningful difference in self-folding free energies of gRNAs across different stability groups (Fig. 7). The gRNAs GC content and $\Delta \mathrm{G}$ of gRNAs were shown to significantly impact the cleavage efficacy (Ma et al., 2015; Jensen et al., 2017). 
309 Our results demonstrated a very weak relationship of these parameters with cleavage

310 efficiencies. However, the significance of the observed relationships could not be established.

311 Further, our results showed no association of $\Delta \mathrm{G}$ of $\operatorname{sgRNAs}$ with the efficacy, which is in

312 agreement with previous findings (Jensen et al., 2017). Interestingly, we also found some gRNAs

313 in the dataset, which once were non-functional and became functional against the same target

314 sites upon swapping and vice versa, indicating that some other extrinsic factors besides sequence

315 and structure features are also working for determining their functionality (Durr et al., 2018).

316 The results of our study demonstrate that the sgRNAs targeting plant ineffective regions are

317 different in various parameters from the sgRNAs designed against protein-coding regions of the

318 mammalian genomes. This indicates the need for designing high throughput CRISPR screening

319 studies considering the ineffective regions in addition to the whole genomic landscape in plants.

320 The difference in sgRNAs activities against plants and mammalians genomes was also

321 demonstrated during the formation of design criteria for efficient sgRNAs prediction using in

322

323

324

325

vivo validated plant sgRNAs targeting different genes across different plants (Liang et al., 2016).

Despite demonstrating different features associated with sgRNAs high on-target cleavage

efficacy against ineffective genomic regions, the experimental validation of these results is required.

326

327

328

329

330

331

332

333

334

335

336

337

338

339

340

341

342

343

344

345

346

347

348

\section{Conclusions}

In conclusion, our study demonstrates the features and parameters governing sgRNAs with high on-target efficacy against otherwise ineffective regions of the A. thaliana genome. Our findings illustrate that the ineffective regions of the genome are equally important to consider while designing sgRNAs prediction models. Moreover, we show that plant sgRNAs targeting various ineffective regions of DNA do not strictly follow the parameters designed for protein-coding regions, which are implemented in various sgRNAs design tools. These results indicate the requirement of designing plant genome wide CRISPR screening studies considering the entire genomic context for the rapid prediction of efficient sgRNAs. In this regard, our study can serve as a paradigm for the comprehensive analysis of hundreds of sgRNAs sequences for inferring highly meaningful and statistically significant features for the development of a cost- and timeefficient plant sgRNAs design tool. The prospects encompass the experimental validation of the outcomes of the study.

\section{Acknowledgements}

Not applicable.

\section{References}

Bae S, Kweon J, Kim HS, Ki JS. 2014. Microhomology-based choice of Cas9 nuclease target sites. Nature Methods 11:705-706 DOI: 10.1038/nmeth.3015.

Barrangou R, Fremaux C, Deveau H, Richards M, Boyaval P, Moineau S, Romero DA, Horvath P. 2007. CRISPR provides acquired resistance against viruses in prokaryotes. Science 315:17091712 DOI: $10.1126 /$ science. 1138140.

Böhmdorfer G, Wierzbicki AT. 2015. Control of chromatin structure by long noncoding RNA. Trends in Cell Biology 25:623-632 DOI: 10.1016/j.tcb.2015.07.002. 
349 Bortesi L, Zhu C, Zischewski J, Perez L, Bassié L, Nadi R, Forni G, Lade SB, Soto E, Jin X, 350 Medina V, Villorbina G, Muñoz P, Ferré G, Fischer R, Twyman RM, Capell T, Christou P, 351 Schillberg S. 2016. Patterns of CRISPR/Cas9 activity in plants, animals and microbes. Plant 352 Biotecnology Journal 14(12): 2203-2216 DOI: 10.1111/pbi.12634.

353 Brazelton VA, Zarecor S, Wright DA, Wang Y, Liu J, Chen K, Yang B, Lawrence-Dill CJ. 2015. A quick guide to CRISPR sgRNA design tools. GM Crops and Food 6:266-276 DOI: 10.1080/21645698.2015.1137690.

356

357 Cao Q, Ma J, Chen C-H, Xu H, Chen Z, Li W, Liu XS. 2017. CRISPR-FOCUS: A web server for designing focused CRISPR screening experiments. PLOS One 12:e0184281 DOI:

358 10.1371/journal.pone.0184281.

359 Chaudhary J, Alisha A, Bhatt V, Chandanshive S, Kumar N, Mir Z, Kumar A, Yadav SK, 360 Shivaraj SM, Sonah H, Deshmukh R. 2019. Mutation Breeding in Tomato: Advances, 361 Applicability and Challenges. Plants 8:128 DOI: 10.3390/plants8050128.

362 Chari R, Mali P, Moosburner M, Church GM. 2015. Unraveling CRISPR-Cas9 genome

363 engineering parameters via a library-on-library approach. Nature Methods 12:823-6 DOI: 10.1038/nmeth.3473.

365 Chari R, Yeo NC, Chavez A, Church GM. 2017, sgRNA Scorer 2.0- a species independent model to predict CRISPR/Cas9 activity. ACS Synthetic Biology 6:902-904 DOI: 10.1021/acssynbio.6b00343.

368 Cram D, Kulkarni M, Buchwaldt M, Rajagopalan N, Bhowmik P, Rozwadowski K, Parkin IAP, 369 Sharpe AG, Kagale S. 2019. WheatCRISPR: a web-based guide RNA design tool for 370 CRISPR/Cas9-mediated genome editing in wheat. BMC Plant Biology 19:474 DOI:

371 10.1186/s12870-019-2097-z.

372 Cong L, Ran FA, Cox D, Lin S, Barretto R, Habib N, Hsu PD, Wu X, Jiang W, Marraffini LA, Zhang F. 2013. Multiplex genome engineering using CRISPR/Cas systems. Science 339:819-823 DOI: $10.1126 /$ science.1231143.

375 Doench JG, Hartenian E, Graham DB, Tothova Z, Hegde M, Smith I, Sullender M, Ebert BL, Xavier RJ, Root DE. 2014. Rational design of highly active sgRNAs for CRISPR-Cas9 mediated gene inactivation. Nature Biotechnology 32:1262-1267 DOI: 10.1038/nbt.3026. Doench JG, Fusi N, Sullender M, Hegde M, Vaimberg EW, Donovan KF, Smith I, Tothova Z, Wilen C, Orchard R, Virgin HW, Listgarten J, Root DE. 2016. Optomized sgRNA design to maximize activity and minimize off-target effects of CRISPR-Cas9. Nature Biotechnology 34:184-191 DOI: 10.1038/nbt.3437.

382 Durr J, Papareddy R, Nakajima K, Gutierrez-Marcos J. 2018. Highly efficient heritable targeted deletions of gene clusters and non-coding regulatory regions in Arabidopsis using CRISPR/Cas9.

384 Scientific Reports 8:4443 DOI: 10.1038/s41598-018-22667-1.

385 Fu Y, Sander JD, Reyon D, Cascio VM, Joung JK. 2014. Improving CRISPR-Cas nuclease specificity using truncated guide RNAs. Nature Biotechnology 32(3):279-84 DOI:

388 Fusi N, Smith I, Doench J, Listgarten J. 2015. In silico predictive modeling of CRISPR/Cas9 389 guide efficiency. bioRxiv DOI: 10.1101/021568.

390 Gilchrist E, Haughn G. 2010. Reverse genetics techniques: engineering loss and gain of gene 391 function in plants. Briefings in Functional Genomics 9:103-110 DOI: 10.1093/bfgp/elp059. 392 Heigwer F, Kerr G, Boutros M. 2014. E-CRISP: fast CRISPR target site identification. Nature 393 Methods 11:122-123 DOI: 10.1038/nmeth.2812. 
394 Hsu PD, Scott DA, Weinstein JA, Ran FA, Konermann S, Agarwala V, Li Y, Fine EJ, Wu X, 395 Shalem O, Cradick TJ, Marraffini LA, Bao G, Zhang F. 2013. DNA targeting specificity of 396 RNA-guided Cas9 nucleases. Nature Biotechnology 31:827-832 DOI: 10.1038/nbt.2647.

397 Hofacker IL. 2003. Vienna RNA secondary structure server. Nucleic Acids Research 31:34293983431 DOI: 10.1093/nar/gkg599.

399 Housden BE, Valvezan AJ, Kelley C, Sopko R, Hu Y, Roesel C, Lin S, Buckner M, Tao R, 400 Yilmazel B, Mohr ES, Manning BD, Perrimon N. 2015. Identification of potential drug targets 401 for tuberous sclerosis complex by synthetic screens combining CRISPR-based knockouts with 402 RNAi. Science Signaling 8:rs9 DOI: 10.1126/scisignal.aab3729.

403 Hussain B, Lucas SJ, Budak H. 2018. CRISPR/Cas9 in plants: at play in the genome and at work 404 for crop improvement. Briefings in Functional Genomics 17(5): 319-328 DOI:

$40510.1093 / \mathrm{bfgp} / \mathrm{ely016.}$

406 Jensen KT, Fløe L, Petersen TS, Huang J, Xu F, Bolund L, Luo Y, Lin L. 2017. Chromatin 407 accessibility and guide sequence structure affect CRISPR-Cas9 gene editing efficiency. FEBS 408 Letters 591:1892-1901 DOI: 10.1002/1873-3468.12707.

409 Jinek M, Chylinski K, Fonfara I, Hauer MH, Doudna JA, Charpentier E. 2012. A Programmable 410 dual-RNA-guided DNA endonuclease in adaptive bacterial immunity. Science 337:816-821 DOI: 411 10.1126/science.1225829.

412 Kim D, Alptekin B, Budak H. 2018. CRISPR/Cas9 genome editing in wheat. Functional and 413 integrative Genomics 18:31-41 DOI: 10.1007/s10142-017-0572-x.

414 Labuhn M, Adams FF, Ng M, Knoess S, Schambach A, Charpentier EM, Schwarzer A, Mateo 415 JL, Klusmann J-H, Heckl D. 2018. Refined sgRNA efficiency prediction improves large- and 416 small-scale CRISPR-Cas9 applications. Nucleic Acids Research 46:1375-1385 DOI:

417 10.1093/nar/gkx1268.

418 Labun K, Montague TG, Krause M, Cleuren YNT, Tjeldnes H, Valen E. 2019. CHOPCHOP v3: 419 expanding the CRISPR web toolbox beyond genome editing. Nucleic Acids Research 47:W171420 W174 DOI: 10.1093/nar/gkz365.

421 Lee YW, Gould BA, Stinchcombe JR. 2014. Identifying the genes underlying quantitative traits: 422 a rationale for the QTN programme. AoB Plants 6:plu004 DOI: 10.1093/aobpla/plu004.

423 Lei Y, Lu L, Liu H-Y, Li S, Xing F, Chen L-L. 2014. CRISPR-P: A web tool for synthetic 424 single-guide RNA design of CRISPR-system in plants. Molecular Plant 7:1494-1496 DOI: $42510.1093 / \mathrm{mp} / \mathrm{ssu} 044$.

426 Li J-F, Norville JE, Aach J, McCormack M, Zhang D, Bush J, Church GM, Sheen J. 2013. 427 Multiplex and homologous recombination-mediated genome editing in Arabidopsis and 428 Nicotiana benthamiana using guide RNA and Cas9. Nature Biotechnology 31:688-691 DOI: 429 10.1038/nbt.2654.

430 Liang G, Zhang H, Lou D, Yu D. 2016. Selection of highly efficient sgRNAs for CRISPR/Cas9431 based plant genome editing. Scientific Reports 6:21451 DOI: 10.1038/srep21451.

432 Liu G, Zhang Y, Zhang T. 2019. Computational approaches for effective CRISPR guide RNA 433 design and evaluation. Computational and Structure Biotechnology Journal 18:35-44 DOI:

434 10.1016/j.csbj.2019.11.006.

435 Liu H, Ding Y, Zhou Y, Jin W, Xie K, Chen L-L. 2017. CRISPR-P 2.0: an improved 436 CRISPR/Cas9 tool for genome editing in plants. Molecular Plant 10:530-532 DOI:

437 10.1016/j.molp.2017.01.003. 
438 Liu X, Homma A, Sayadi J, Yang S, Ohashi J, Takumi T. 2016. Sequence features associated 439 with the cleavage efficiency of CRISPR/Cas9 system. Scientific Reports 6:19675 DOI:

$440 \quad 10.1038 /$ srep 19675.

441 Lorenz R, Luntzer D, Hofacker IL, Stadler PF, Wolfinger MT. 2016. SHAPE directed RNA

442 folding. Bioinformatics 32:145-7 DOI:10.1093/bioinformatics/btv523.

443 Ma X, Zhang Q, Zhu Q, Liu W, Chen Y, Qiu R, Wang B, Yang Z, Li H, Lin Y, Xie Y, Shen R, 444 Chen S, Wang Z, Chen Y, Guo J, Chen L, Zhao X, Dong Z, Liu Y-G. 2015. A robust

445 CRISPR/Cas9 system for convenient high-efficiency multiplex genome editing in monocot and 446 dicot plants. Molecular Plant 8:1274-84 DOI: 10.1016/j.molp.2015.04.007.

447 Mali P, Aach J, Stranges PB, Esvelt KM, Moosburner M, Kosuri S, Yang L, Church GM. 2013.

448 CAS9 transcriptional activators for target specificity screening and paired nikases for

449 cooperative genome engineering. Nature Biotechnology 31:833-8 DOI: 10.1038/nbt.2675.

450 McCaskill JS. 1990. The equilibrium partition function and base pair binding probabilities for

451 RNA secondary structure. Biopolymers 29:1105-19 DOI: 10.1002/bip.360290621.

452 Mendoza BJ, Trinh CT. 2018. Enhanced guide-RNA design and targeting analysis for precise

453 CRISPR genome editing of single and consortia of industrially relevant and non-model

454 organisms. Bioinformatics 34:16-23 DOI: 10.1093/bioinformatics/btx564.

455 Minkenberg B, Zhang J, Xie K, Yang Y. 2018. CRISPR-PLANT v2: an online resource for

456 highly specific guide RNA spacers based on improved off-target analysis. Plant Biotechnology

457 17:5-8 DOI: 10.1111/pbi.13025.

458 Moreno-Mateos MA, Vejnar CE, Beaudoin J-D, Fernandez JP, Mis KE, Khokha MK, Giraldez

459 AJ. 2015. CRISPRscan: designing highly efficient sgRNAs for CRISPR-Cas9 targeting in vivo.

460 Nature Methods 12:982-8 DOI: 10.1038/nmeth.3543.

461 Naim F, Shand K, Hayashi S, O’Brien M, McGree J, Johnson AAT, Dugdale B, Waterhouse

462 PM. 2020. Are the current gRNA ranking prediction algorithms useful for genome editing in

463 plants? PLOS One 15:e0227994 DOI: 10.1371/journal.pone.0227994.

464 Nekrasov V, Staskawicz B, Weigel D, Jones JD, Kamoun S. 2013. Targeted mutagenesis in the

465 model plant Nicotiana benthamiana using Cas9 RNA-guided endonuclease. Nature Biotechnology

466 31:691-693 DOI: 10.1038/nbt.2655.

467 Oladosu Y, Rafii MY, Abdullah N, Hussin G, Ramli A, Rahim HA, Miah G, Usman M. 2016.

468 Principle and application of plant mutagenesis in crop improvement: a review. Biotechnology

469 and Biotechnological Equipment 30:1-16 DOI: 10.1080/13102818.2015.1087333.

470 Pattanayak V, Lin S, Guilinger JP, Ma E, Doudna JA, Liu D.R. 2013. High-throughput profiling

471 of off-target DNA cleavage reveals RNA-programmed Cas9 nuclease specificity. Nature

472 Biotechnology 31:839-843 DOI: 10.1038/nbt.2673.

473 Ran FA, Hsu PD, Lin C-Y, Gootenberg JS, Konermann S, Trevino AE, Scott DA, Inoue A,

474 Matoba S, Zhang Y, Zhang F. 2013. Double nicking by RNA-guided CRISPR Cas9 for enhanced

475 genome editing specificity. Cell 154:1380-9 DOI: 10.1016/j.cell.2013.08.021.

476 Ren X, Yang Z, Xy J, Sun J, Mao D, Hu Y, Yang S-J, Qiao H-H, Wang X, Hu Q, Deng P, Liu L-

477 P, Ji J-Y, Li JB, Ni J-Q. 2014. Enhanced specificity and efficiency of the CRISPR/Cas9 system

478 with optimized sgRNAs parameters in Drosophila. Cell Reports 9(3):1151-1162 DOI:

479 10.1016/j.celrep.2014.09.004.

480 Rinaldo AR, Ayliffe M. 2015. Gene targeting and editing in crop plants: a new era of precision

481 opportunities. Molecular Breeding 35:40 DOI: 10.1007/s11032-015-0210-z.

482 Sander JD, Joung JK. 2014. CRISPR-Cas systems for editing, regulation and targeting genomes.

483 Nature Biotechnology 32:347-355 DOI: 10.1038/nbt.2842.

Peer) reviewing PDF | (2020:10:53534:2:0:NEW 8 Mar 2021) 
484 Shalem O, Sanjana NE, Zhang F. 2015. High-throughput functional genomics using CRISPR485 Cas9. Nature Reviews Genetics 16:299-311 DOI: 10.1038/nrg3899.

486 Shan Q, Wang Y, Li J, Zhang Y, Chen K, Liang Z, Zhang K, Liu J, Xi JJ, Qiu JL, Gao C. 2013. 487 Targeted genome modification of crop plants using a CRISPR-Cas system. Nature

488 Biotechnology 31:686-688 DOI: 10.1038/nbt.2650.

489 Shanmugam A, Nagarajan A, Pramanayagam S. 2017. Non-coding DNA- a brief review. Journal 490 of Applied Biology and Biotechnology 5:42-47 DOI: 10.7324/JABB.2017.50507.

491 Tadege M, Wang TL, Wen J, Ratet P, Mysore KS. 2009. Mutagenesis and beyond! Tools for 492 understanding legume biology. Plant Physiology 151:978-984 DOI: 10.1104/pp.109.144097.

493 Thyme SB, Akhmetova L, Montague TG, Valen E, Schier AF. 2016. Internal guide RNA 494 interactions interfere with Cas9-mediated cleavage. Nature Communications 7:11750 DOI: $49510.1038 /$ ncomms 11750.

496 Wang T, Wei JJ, Sabatini DM, Lander ES. 2014. Genetic screens in human cells using CRISPR497 Cas9 system. Science 343:80-4 DOI: 10.1126/science.1246981.

498 Wiedenheft, B., Sternberg, S.H., Doudna, J.A., 2012. RNA-guided genetic silencing systems in 499 bacteria and archaea. Nature 482:331-338 DOI: 10.1038/nature10886.

500 Wong N, Liu W, Wang X. 2015. WU-CRISPR: characteristics of functional guide RNAs for the 501 CRISPR/Cas9 system. Genome Biology 16:218 DOI: 10.1186/s13059-015-0784-0.

502 Wu J-L, Wu C, Lei C, Baraoidan M, Bordeos A, Madamba MRS, Ramos-Pamplona M, Mauleon 503 R, Portugal A, Ulat VJ, Bruskiewich R, Wang G, Leach J, Khush G, Leung H. 2005. Chemical504 and irradiation-induced mutants of indica rice IR64 for forward and reverse genetics. Plant 505 Molecular Biology 59:85-97 DOI: 10.1007/s11103-004-5112-0.

506 Wu R, Lucke M, Jang Y-T, Zhu W, Symeonidi E, Wang C, Fitz J, Xi W, Schwab R, Weigel D. 507 2018. An efficient CRISPR vector toolbox for engineering large deletions in Arabidopsis 508 thaliana. Plant Methods 14:65 DOI: 10.1186/s13007-018-0330-7.

509 Wu X, Scott DA, Kriz AJ, Chiu AC, Hsu PD, Dadon DB, Cheng AW, Trevino AE, Konermann 510 S, Chen S, Jaenisch R, Zhang F, Sharp PA. 2014. Genome-wide binding of the CRISPR 511 endonuclease Cas9 in mammalian cells. Nature Biotechnology 32:670-6 DOI: 10.1038/nbt.2889.

512 Xie K, Yang Y. 2013. RNA-guided genome editing in plants using a CRISPR-Cas system.

513 Molecular Plant 6:1975-1983 DOI: 10.1093/mp/sst119.

514 Xie K, Zhang J, Yang Y. 2014. Genome-wide prediction of highly specific guide RNA spacers

515 for the CRISPR-Cas9-mediated genome editing in model plants and major crops. Molecular

516 Plant 7:923-6 DOI: 10.1093/mp/ssu009.

517 Xie X, Ma X, Zhu Q, Zeng D, Li G, Liu Y-G. 2017. CRISPR-GE: A convenient software toolkit 518 for CRISPR-based genome editing. Molecular Plant 10:1246-1249 DOI:

519 10.1016/j.molp.2017.06.004.

520 Xu H, Xiao T, Chen C-H, Li W, Meyer CA, Wu Q, Wu D, Cong L, Zhang F, Liu JS, Brown M, 521 Liu XS. 2015. Sequence determinants of improved CRISPR sgRNA design. Genome Research 522 25:1147-57 DOI: 10.1101/gr.191452.115.

523 Zuker M, Stiegler P. 1981. Optimal computer folding of large RNA sequences using 524 thermodynamics and auxiliary information. Nucleic Acids Research 9:133-148 DOI:

$52510.1093 /$ nar/9.1.133. 


\section{Figure 1}

The schematic demonstration of the sgRNA secondary structure.

The figure shows that the sgRNA consists of two main parts i.e., gRNA and tracrRNA that are connected through a hairpin-like structure. The tracrRNA and the hairpin-like structure constitute the scaffolding region of the sgRNA. The presence of $\mathrm{N}$ (red colour) at $5^{\prime}$ end denotes 20 bp long gRNA sequence that base pairs with the complementary sequence of target DNA (green colour). The bold black colour represents the PAM site that is present adjacent to the target site. Moreover, the secondary structure of sgRNA is characterised by the presence of several secondary structural elements like stem loop RAR (blue colour), stem loop 1 (orange colour), stem loop 2 (pink colour), and stem loop 3 (cyan colour). The last three bases (bold red) of gRNA and the first three bases of tracrRNA (bold purple) mark important nucleotides. The solid lines represent Waston-Crick base pairing, while dashed lines depict non-Waston-Crick or Wobble base pairing. 


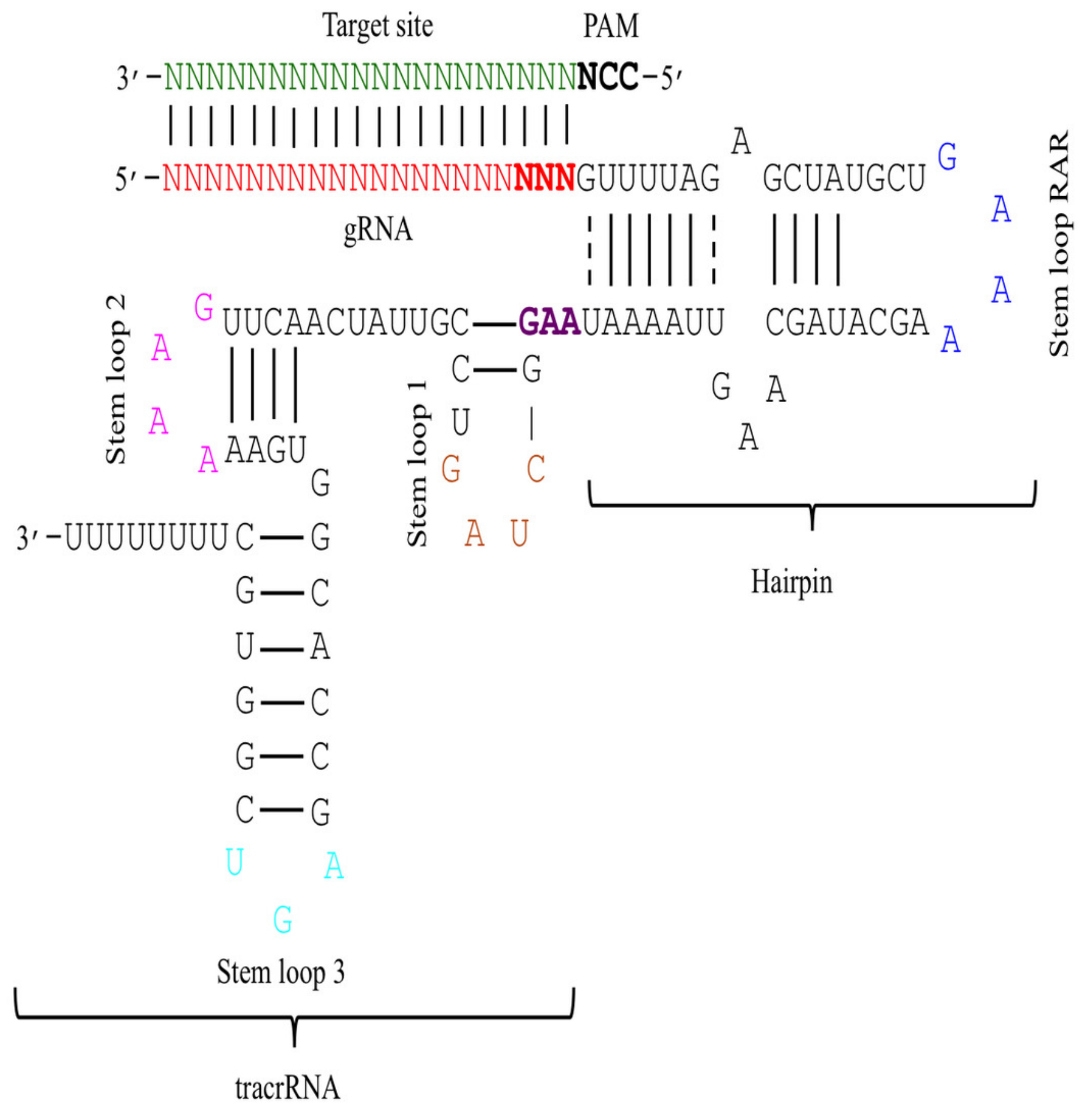


Figure 2

Sequence logo describing nucleotide preferences in gRNAs targeting ineffective DNA regions.

The figure represents the logo of nucleotide preferences in gRNA sequences and the height of the nucleotide describes its frequency of occurrence at a particular position.

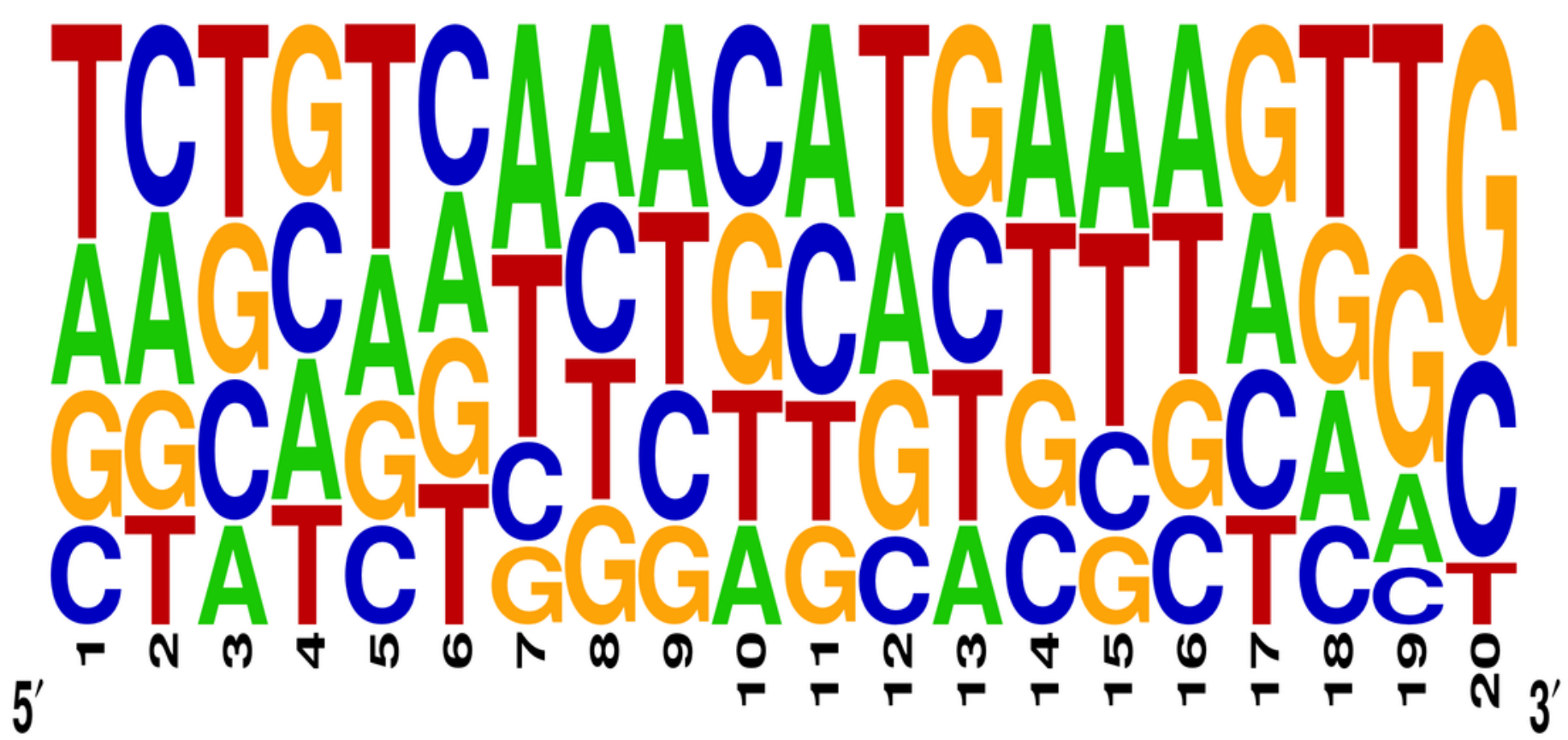




\section{Figure 3}

Correlation of GC content and cleavage efficacy.

(A) Analysis of gRNAs full-length GC content and cleavage efficacy. No significant difference overall. (B) The GC content of the PAM proximal seed region (1-12nt) significantly affects the cleavage efficacy. (C) No significant effect of GC content on efficacy within PAM distal region (13-20 nt). Kruskal-Wallis tests are indicated. ns and *: indicate non-significant and significant at $5 \%$ probability level.

A

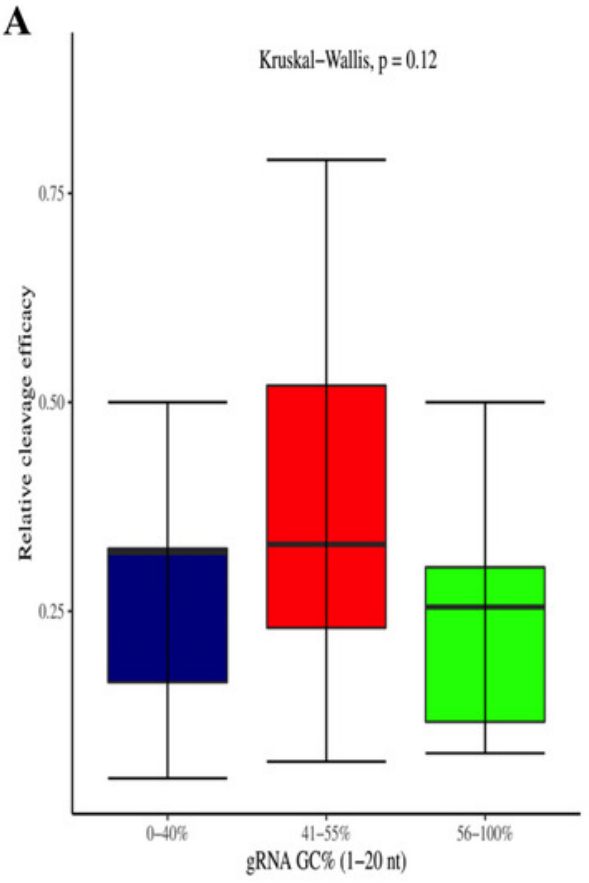

B

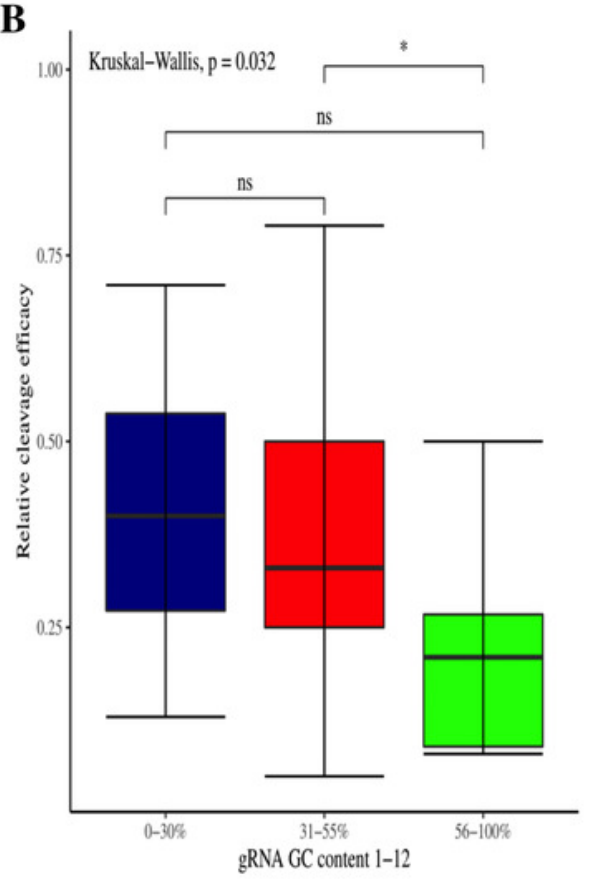

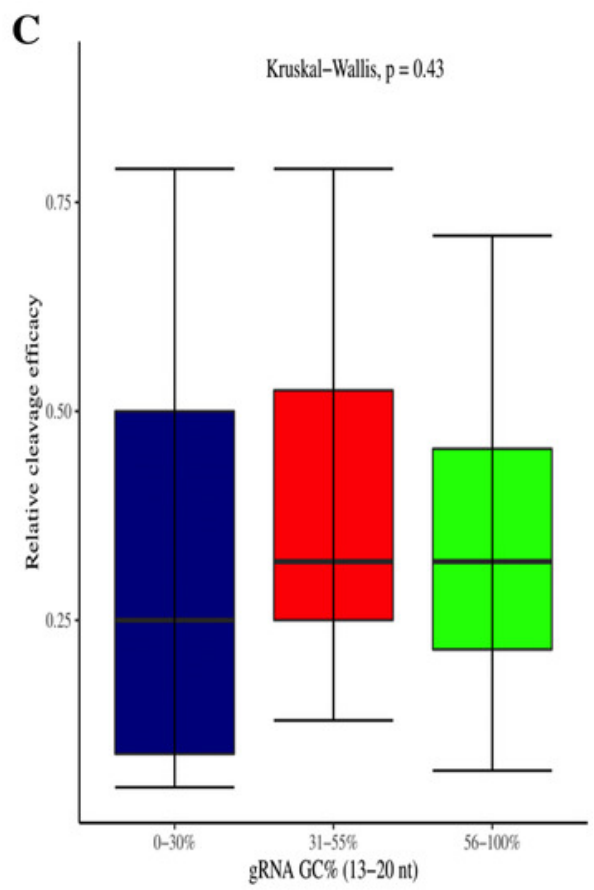


Figure 4

Analysis of same dinucleosides impact on cleavage efficacy.

(A-D) Overall no significant effect of same dinucleosides on cleavage efficacy. $0=$ gRNAs without dinucleosides, $1=$ gRNAs with one dinucleoside and $2=$ gRNAs with two dinucleosides. The level of significance is tested with the Kruskal-Wallis test.
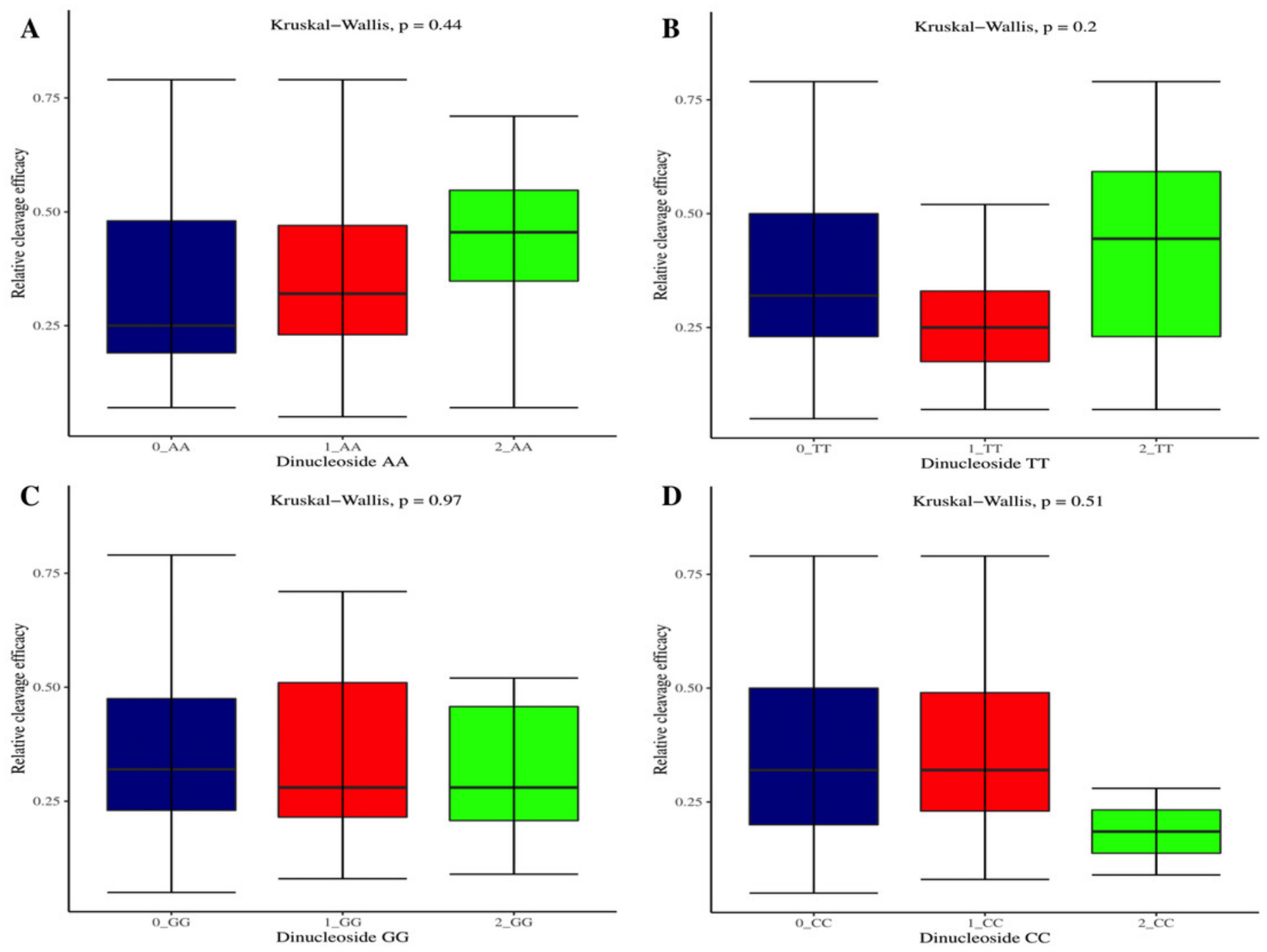


\section{Figure 5}

The correlation of same trinucleosides with cleavage efficacy.

(A-D) The Wilcoxon test shows no significant impact of same trinucleosides on sgRNAs activity. $0=$ gRNAs without trinucleoside repeats and $1=$ gRNAs with one trinucleoside repeat.
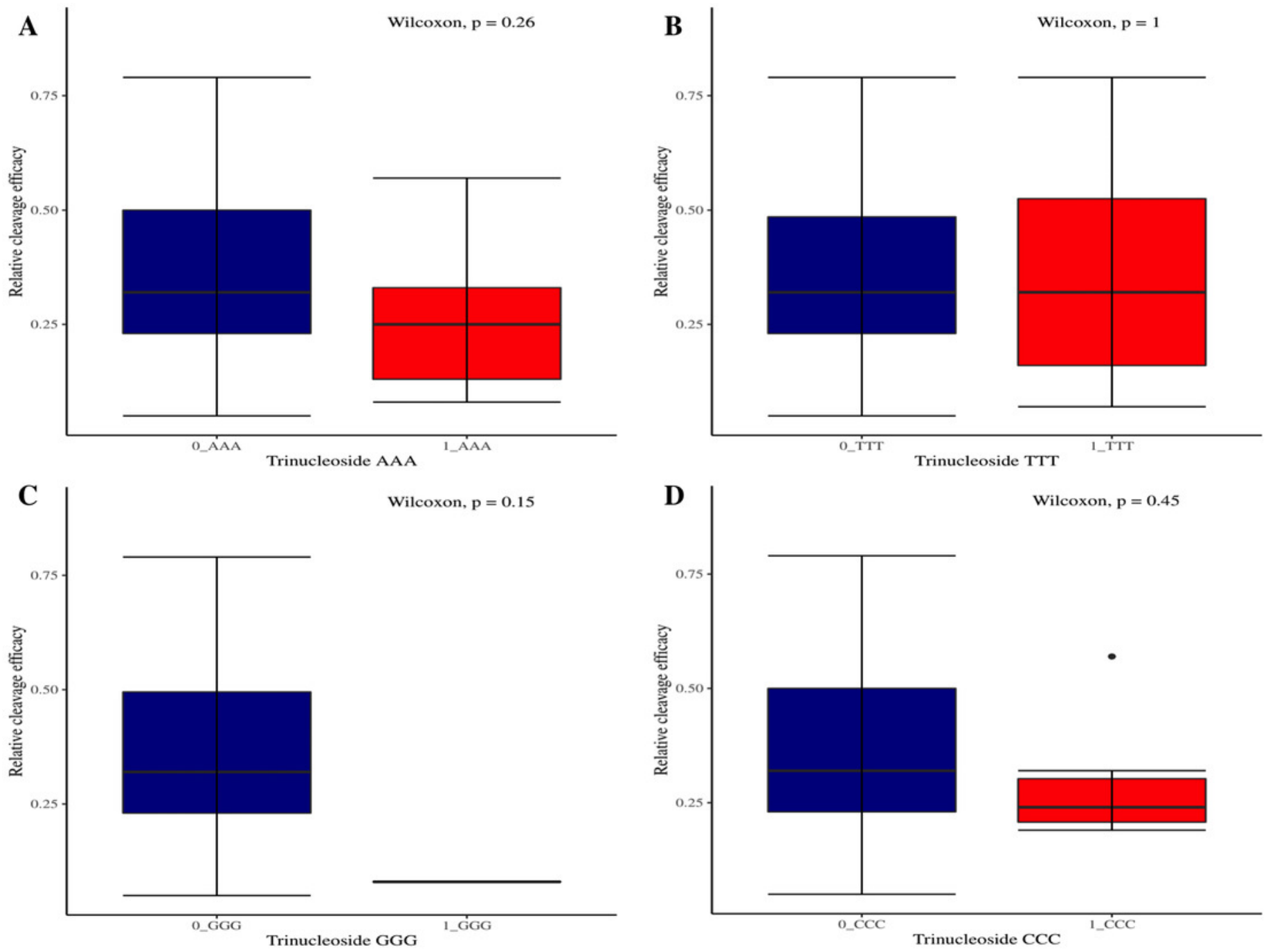


\section{Figure 6}

Analysis of variable nucleotide and target DNA strand impact on cleavage efficacy.

(A) Although no overall significant difference in the usage of PAM variable nucleotides on efficacy is ascertained as indicated by Kruskal-Wallis test. However, the variable nucleotides $T(0.3968)$ and $A(0.3031)$ show better cleavage efficacy than $G(0.266)$ and $C(0.165)$. (B) No overall significant difference as indicated by the Wilcoxon test, however gRNAs targeting transcribed strand show better efficacy compared to those targeting non-transcribed strand.

A

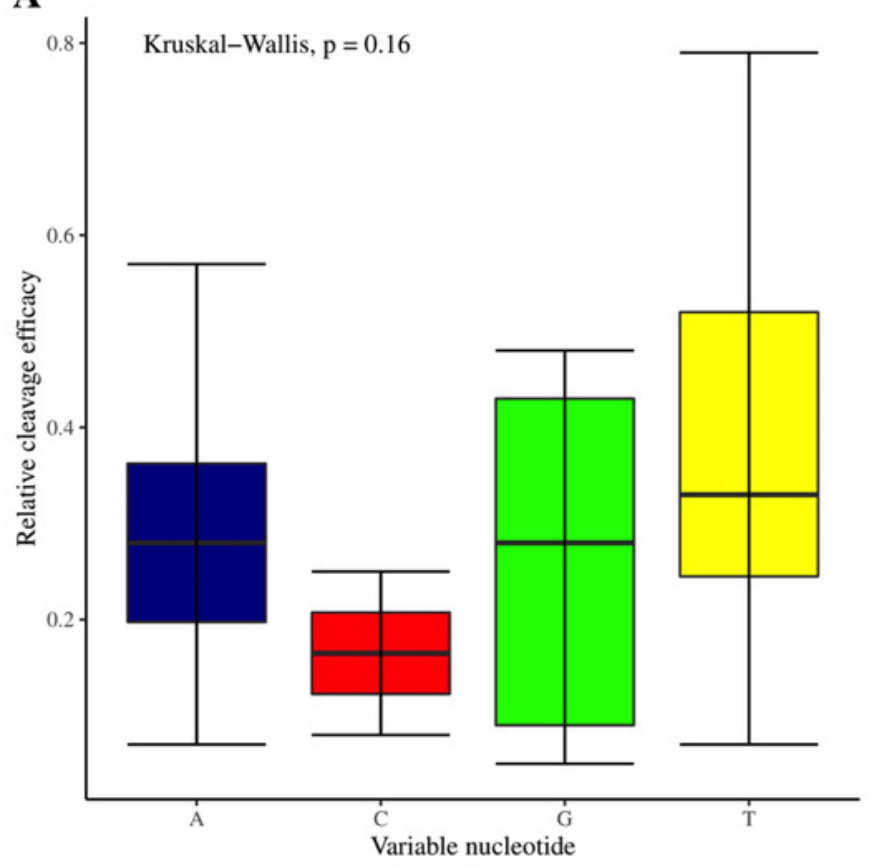

B

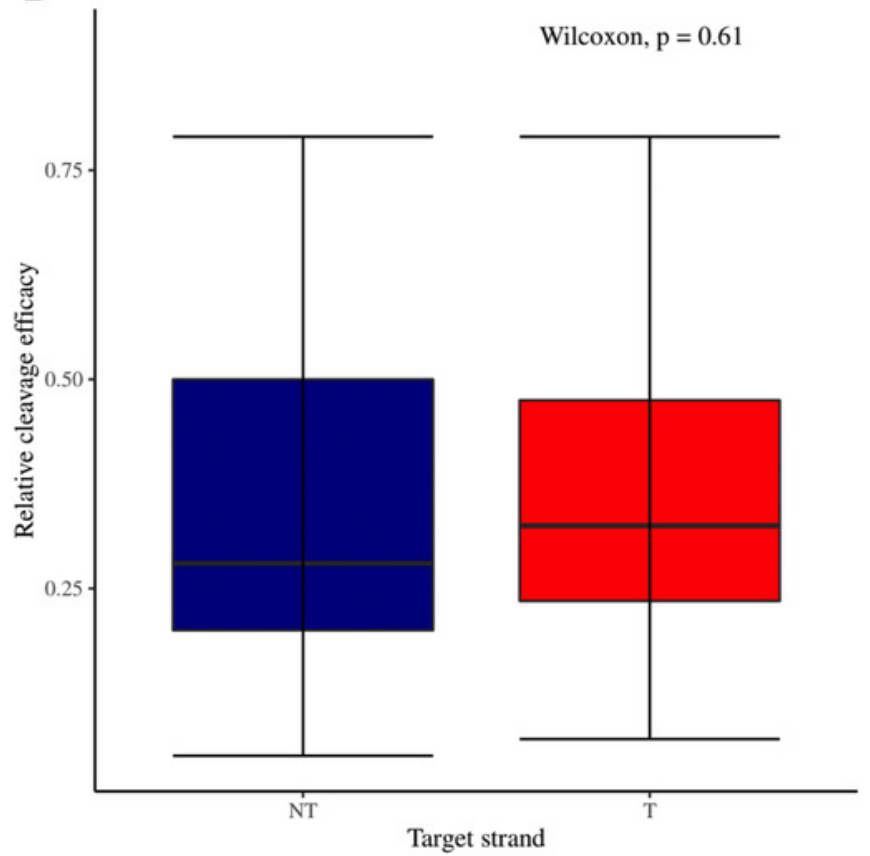


Figure 7

Effect of gRNAs self-folding free energies $(\Delta G)$ on cleavage efficacy.

The Kruskal-Wallis test shows no significant impact of gRNA $\Delta G$ on efficacy overall, however gRNAs with $\Delta \mathrm{G}$ values 0 demonstrate better efficacy.

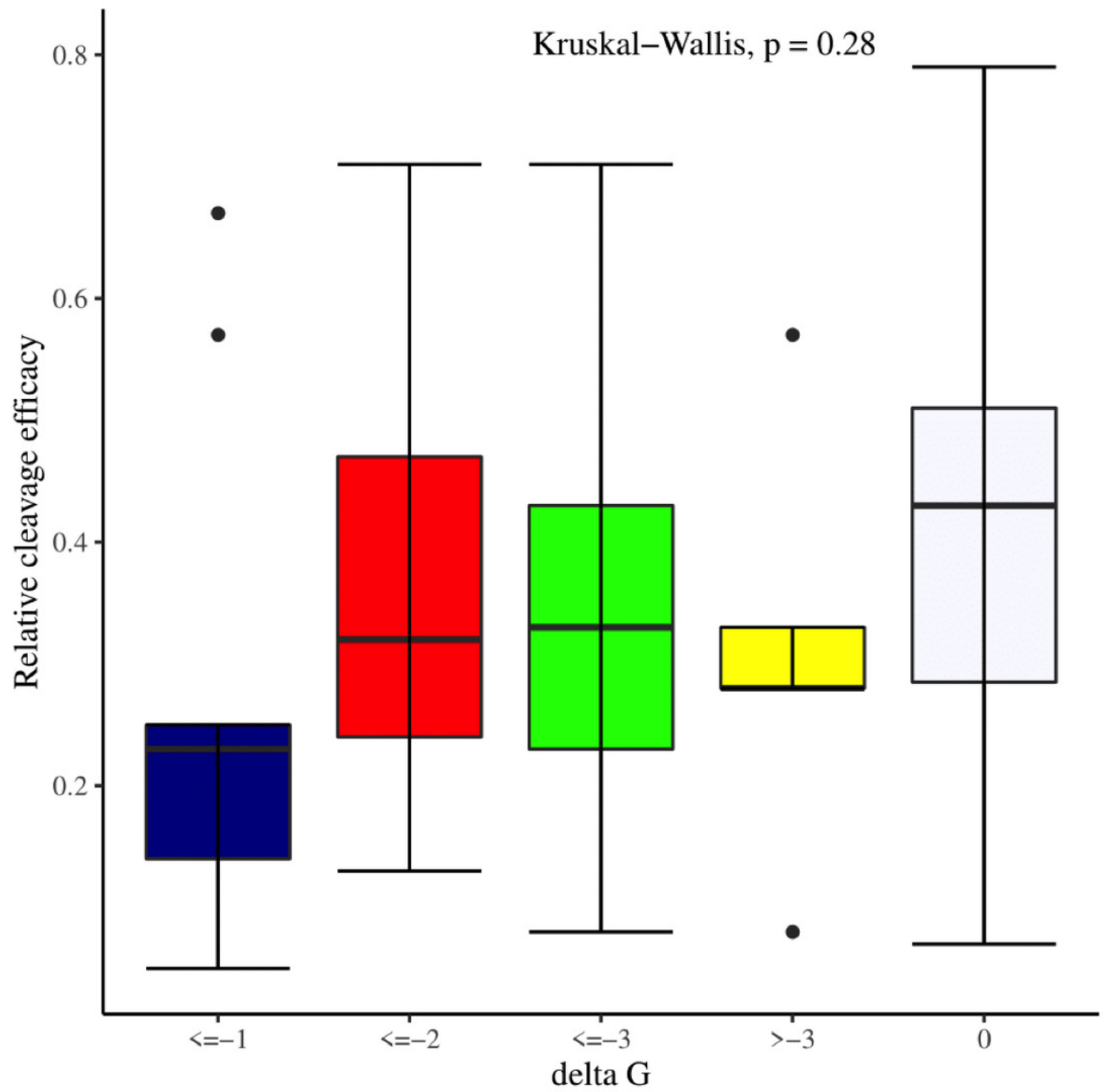


Table $\mathbf{1}$ (on next page)

Different plant-specific computational tools for the prediction of sgRNAs. 


\begin{tabular}{|c|c|c|c|c|c|}
\hline $\begin{array}{l}\text { Computa } \\
\text { tional } \\
\text { Tool }\end{array}$ & Organism & $\begin{array}{l}\text { Off-target } \\
\text { prediction/model } \\
\text { or scoring system }\end{array}$ & $\begin{array}{l}\text { Cleavage } \\
\text { efficacy/mod } \\
\text { el or scoring } \\
\text { system }\end{array}$ & $\begin{array}{l}\text { Web server } \\
\text { address }\end{array}$ & Reference \\
\hline $\begin{array}{l}\text { CRISPR- } \\
\text { PLNAT } \\
\text { Version1 }\end{array}$ & Plants & $\begin{array}{l}\text { Yes/ Hsu et al., } \\
\text { 2013; Mali et al., } \\
\text { 2013; Pattanayak et } \\
\text { al., 2013; Li et al., } \\
\text { 2013; Nekrasov et } \\
\text { al., 2013; Shan et } \\
\text { al., 2013; Xie } \\
\text { \&Yang, } 2013\end{array}$ & No & $\frac{\underline{\text { www.geno }}}{\frac{\text { me.arizona. }}{\text { edu/ceispr }}}$ & $\begin{array}{l}\text { Xie, Zhang \& } \\
\text { Yang, } 2014 \text { (doi: } \\
\text { 10.1093/mp/ssu00 } \\
\text { 9) }\end{array}$ \\
\hline $\begin{array}{l}\text { CRISPR- } \\
\text { PLNAT } \\
\text { Version2 }\end{array}$ & Plants & $\begin{array}{l}\text { Yes/Minkenberg et } \\
\text { al., } 2018\end{array}$ & No & $\begin{array}{l}\frac{\text { www.geno }}{\text { me.arizona. }} \\
\underline{\text { edu/ceispr } 2 /}\end{array}$ & $\begin{array}{l}\text { Minkenberg et al., } \\
2018 \text { (doi: } \\
10.1111 / \text { pbi. } 1302 \\
\text { 5) }\end{array}$ \\
\hline $\begin{array}{l}\text { CRISPR- } \\
\text { P }\end{array}$ & Plants & $\begin{array}{l}\text { Yes/Hsu et al., } \\
2013\end{array}$ & No & $\begin{array}{l}\underline{\text { http://cbi.hz }} \\
\underline{\text { au.edu.cn/cr }} \\
\underline{\text { ispr/ }}\end{array}$ & $\begin{array}{l}\text { Lei et al., } 2014 \\
\text { (doi: } \\
\text { 10.1093/mp/ssu04 } \\
\text { 4) }\end{array}$ \\
\hline $\begin{array}{l}\text { CRISPR- } \\
\text { P } 2.0\end{array}$ & Plants & $\begin{array}{l}\text { Yes/Doench et al., } \\
2016\end{array}$ & $\begin{array}{l}\text { Yes/Doench } \\
\text { et al., 2014; } \\
\text { Bae et al., } \\
\text { 2014; Ren et } \\
\text { al., 2014; } \\
\text { Liang et al., } \\
\text { 2016; Lorenz } \\
\text { et al., } 2016 \\
\end{array}$ & $\begin{array}{l}\frac{\text { http://cbi.hz }}{\text { au.edu.cn/cr }} \\
\underline{\text { ispr2/ }}\end{array}$ & $\begin{array}{l}\text { Liu et al., } 2017 \\
\text { (doi: } \\
\text { 10.1016/j.molp.20 } \\
\text { 17.01.003) }\end{array}$ \\
\hline CGAT & Plants & $\begin{array}{l}\text { Yes/sequence } \\
\text { identity }\end{array}$ & $\begin{array}{l}\text { Yes/Ren et } \\
\text { al., } 2014\end{array}$ & $\begin{array}{l}\frac{\text { http://cropbi }}{\text { oengineerin }} \\
\text { g.iastate.edu } \\
\text { /cgat }\end{array}$ & $\begin{array}{l}\text { Brazeltonet al., } \\
2015 \text { (doi: } \\
10.1080 / 2164569 \\
8.2015 .1137690)\end{array}$ \\
\hline $\begin{array}{l}\text { CRISPR- } \\
\text { GE }\end{array}$ & $\begin{array}{l}\text { Plant and } \\
\text { non-plant } \\
\text { organisms }\end{array}$ & $\begin{array}{l}\text { Yes/Doench et al, } \\
2016\end{array}$ & $\begin{array}{l}\text { Yes/Ma et } \\
\text { al., } 2015\end{array}$ & $\begin{array}{l}\text { http://skl.sc } \\
\text { au.edu.cn/ }\end{array}$ & $\begin{array}{l}\text { Xie et al., } 2017 \\
\text { (doi: } \\
\text { 10.1016/j.molp.20 } \\
\text { 17.06.004) }\end{array}$ \\
\hline $\begin{array}{l}\text { WheatC } \\
\text { RISPR }\end{array}$ & Wheat & $\begin{array}{l}\text { Yes/Doench et al., } \\
2016\end{array}$ & $\begin{array}{l}\text { Yes/Doench } \\
\text { et al., } 2016\end{array}$ & $\begin{array}{l}\frac{\text { http://crispr. }}{\text { bioinfo.nrc. }} \\
\text { ca/WheatCr } \\
\underline{\text { ispr/ }}\end{array}$ & $\begin{array}{l}\text { Cram et al., } 2019 \\
\text { (doi: } \\
10.1186 / \mathrm{s} 12870- \\
019-2097-z \text { ) }\end{array}$ \\
\hline
\end{tabular}




\section{Table 2 (on next page)}

Frequency table showing nucleotide base frequencies at each position of gRNAs. 
1

\begin{tabular}{|c|c|c|c|c|c|}
\hline Base position & & TAs & bas & ncy & $p$-value \\
\hline & $\mathbf{A}$ & $\mathbf{G}$ & $\mathbf{C}$ & $\mathbf{T}$ & \\
\hline 1 & 14 & 13 & 10 & 21 & $5.2 \mathrm{E}-01$ \\
\hline 2 & 17 & 12 & 18 & 11 & $2.3 \mathrm{E}-01$ \\
\hline 3 & 10 & 15 & 14 & 19 & $5.0 \mathrm{E}-01$ \\
\hline 4 & 14 & 17 & 15 & 12 & $5.5 \mathrm{E}-01$ \\
\hline 5 & 14 & 12 & 10 & 22 & $3.6 \mathrm{E}-01$ \\
\hline 6 & 14 & 14 & 16 & 14 & 7.7E-01 \\
\hline 7 & 22 & 8 & 10 & 18 & 8.3E-02 \\
\hline 8 & 17 & 12 & 15 & 14 & $7.5 \mathrm{E}-01$ \\
\hline 9 & 18 & 10 & 13 & 17 & $6.2 \mathrm{E}-01$ \\
\hline 10 & 10 & 17 & 18 & 13 & $1.7 \mathrm{E}-01$ \\
\hline 11 & 19 & 10 & 17 & 12 & $2.0 \mathrm{E}-01$ \\
\hline 12 & 16 & 15 & 9 & 18 & $6.8 \mathrm{E}-01$ \\
\hline 13 & 10 & 18 & 15 & 15 & $3.5 \mathrm{E}-01$ \\
\hline 14 & 19 & 13 & 11 & 15 & $6.9 \mathrm{E}-01$ \\
\hline 15 & 20 & 9 & 10 & 19 & $2.0 \mathrm{E}-01$ \\
\hline 16 & 18 & 13 & 11 & 16 & $8.2 \mathrm{E}-01$ \\
\hline 17 & 15 & 18 & 14 & 11 & $4.1 \mathrm{E}-01$ \\
\hline 18 & 13 & 16 & 10 & 19 & $6.4 \mathrm{E}-01$ \\
\hline 19 & 9 & 21 & 6 & 22 & $8.6 \mathrm{E}-03$ \\
\hline 20 & 0 & 5 & 3 & 1 & $6.4 \mathrm{E}-02$ \\
\hline
\end{tabular}




\section{Table 3(on next page)}

The significantly free accessible nucleotides in the seed region and tracrRNA region of the sgRNAs. 
1

\begin{tabular}{|l|l|l|l|l|l|l|}
\hline & \multicolumn{3}{|c|}{ Seed region } & \multicolumn{5}{c|}{ tracrRNA region } \\
\hline $\begin{array}{l}\text { Nucleotide } \\
\text { position }\end{array}$ & 19 & 20 & 59 & 60 & 61 & 62 \\
\hline$p$-value & $6.3 \mathrm{E}-06$ & $2.4 \mathrm{E}-03$ & $4.0 \mathrm{E}-04$ & $3.5 \mathrm{E}-06$ & $1.7 \mathrm{E}-05$ & $5.3 \mathrm{E}-04$ \\
\hline
\end{tabular}

*Chi-Square test analysis was performed for the inference of $p$-values.

2 